\title{
Conjunctivitis, CTCAE
}

National Cancer Institute

\section{Source}

National Cancer Institute. Conjunctivitis, CT CAE. NCI Thesaurus. Code C143384.

A disorder characterized by inflammation, swelling and redness to the conjunctiva of the eye. 